\title{
High Efficiency Video Coding (HEVC)-Based Surgical Telementoring System Using Shallow Convolutional Neural Network
}

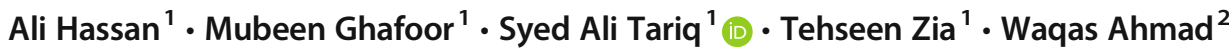 \\ Published online: 12 April 2019 \\ (C) Society for Imaging Informatics in Medicine 2019
}

\begin{abstract}
Surgical telementoring systems have gained lots of interest, especially in remote locations. However, bandwidth constraint has been the primary bottleneck for efficient telementoring systems. This study aims to establish an efficient surgical telementoring system, where the qualified surgeon (mentor) provides real-time guidance and technical assistance for surgical procedures to the on-spot physician (surgeon). High Efficiency Video Coding (HEVC/H.265)-based video compression has shown promising results for telementoring applications. However, there is a trade-off between the bandwidth resources required for video transmission and quality of video received by the remote surgeon. In order to efficiently compress and transmit real-time surgical videos, a hybrid lossless-lossy approach is proposed where surgical incision region is coded in high quality whereas the background region is coded in low quality based on distance from the surgical incision region. For surgical incision region extraction, state-of-the-art deep learning (DL) architectures for semantic segmentation can be used. However, the computational complexity of these architectures is high resulting in large training and inference times. For telementoring systems, encoding time is crucial; therefore, very deep architectures are not suitable for surgical incision extraction. In this study, we propose a shallow convolutional neural network (S-CNN)-based segmentation approach that consists of encoder network only for surgical region extraction. The segmentation performance of S-CNN is compared with one of the state-of-the-art image segmentation networks (SegNet), and results demonstrate the effectiveness of the proposed network. The proposed telementoring system is efficient and explicitly considers the physiological nature of the human visual system to encode the video by providing good overall visual impact in the location of surgery. The results of the proposed S-CNN-based segmentation demonstrated a pixel accuracy of $97 \%$ and a mean intersection over union accuracy of 79\%. Similarly, HEVC experimental results showed that the proposed surgical region-based encoding scheme achieved an average bitrate reduction of $88.8 \%$ at high-quality settings in comparison with default full-frame HEVC encoding. The average gain in encoding performance (signal-to-noise) of the proposed algorithm is $11.5 \mathrm{~dB}$ in the surgical region. The bitrate saving and visual quality of the proposed optimal bit allocation scheme are compared with the mean shift segmentation-based coding scheme for fair comparison. The results show that the proposed scheme maintains high visual quality in surgical incision region along with achieving good bitrate saving. Based on comparison and results, the proposed encoding algorithm can be considered as an efficient and effective solution for surgical telementoring systems for low-bandwidth networks.
\end{abstract}

Keywords Convolutional neural network $(\mathrm{CNN}) \cdot$ Deep learning $(\mathrm{DL}) \cdot \mathrm{HEVC} \cdot$ Medical imaging $\cdot$ Segmentation $\cdot$ Telementoring

\section{Introduction}

In disaster-affected and distant rural areas, there exists a shortage of qualified surgeons. The different surgical procedures

Syed Ali Tariq

s.alitariq1@gmail.com

1 Department of Computer Science, COMSATS University, Islamabad, Pakistan

2 Department of Information Systems and Technology, Mid Sweden University, Sundsvall, Sweden and possibilities that can arise can be difficult to handle by inexperienced surgeons. Surgical telementoring systems can be adopted in such areas to provide real-time technical guidance for surgical procedures, emergency care, surgical education, and health care by experienced remote surgeons [1-3]. One of the significant issues in adopting surgical telementoring systems is the lack of high bandwidth resources in remote areas. The surgical telementoring application requires a unique challenge in the sense that the system should retain the region of interest in nearly lossless quality under constrained bitrate scenario. Rayman et al. [4] proposed that 4-6-Mbps bandwidth is required for good quality 
telementoring applications. In remote or disaster-stricken areas, such high bandwidth resources are unlikely to be available. Therefore, adaptive compression can be useful in surgical telementoring applications where the quality of video varies depending on the nature of the region and the available bandwidth resources. To develop an efficient telementoring system, surgical videos must be compressed such that they can be transmitted over limited bandwidth networks while maintaining high visual quality in critical regions of the video.

One of the most commonly used video compression standards is High Efficiency Video Coding (HEVC) or H.265 [5]. The first version of HEVC was developed by Joint Collaborative Team on Video Coding (JCT-VC), a collaboration between ISO/IEC Moving Pictures Experts Group (MPEG) and ITU-T Visual Coding Experts Group (VCEG) in June 2013. HEVC/H.265 version 4 was approved as an ITU-T standard in December 2016. It offers efficient video compression while maintaining the same video quality at a lower bitrate as compared with its predecessors (MPEG-4 or H.264) [6-8]. Efficient video coding can be utilized in various applications and fields such as mobile video recording [9], video watermarking [10], medical video communications [11-13], medical imaging [14-18], telemedicine [19], rate control optimization [20], lossless video coding [21], high dynamic range video compression [22], and many others. HEVC can be used as part of a video compression tool for surgical telementoring system.

For adaptive compression and maintaining high quality in critical regions, surgical videos can be classified into two segments; the surgical region (SR) and the background region as shown in Fig. 1. For remote surgeon supervising a procedure, the video quality of the SR must be near to lossless, and background region can be of low quality. SR-based video coding can serve as a potential solution for reducing video bitrate and efficiently transmitting high-quality surgical videos over low bandwidth channels.
Image segmentation techniques can be used to identify the SRs in surgical videos. Some common segmentation techniques are mean shift, $k$-means, expectation and maximization, and density-based spatial clustering of application with noise and fuzzy c-means [23, 24]. The mean shift (MST) [25] segmentation is widely used in the field of medical images, i.e., wound segmentation and its boundary determination [26-29]. Such techniques are sensitive to noise and are based on predefined criteria (like intensity information or edges in the image) [23]. These conventional computer vision and machine learning algorithms are very limited in their ability to process large amounts of image data with multiple levels of abstraction and require a lot of manual tuning for each input image.

Recently, deep convolutional neural networks $(\mathrm{CNN})$ have been used as a valuable tool for image segmentation tasks in computer vision applications [30-36]. CNN can be utilized to tackle the problem of multiple levels of abstraction as well as noises and irregularities in images [23, 24]. Some of the popular deep convolutional networks are VGGNet [37], GoogleNet [38], ResNet [39], UNet [40], and SegNet [41]. Although these networks can be used for image segmentation tasks, they are computationally expensive since the network depth (stacked layers) is of crucial importance. The VGGNet [37] consists of 19 layers, GoogleNet [38] consists of 22 layers, ResNet [39] consists of 50 layers, UNet [40] consists of 23 convolutional layers, and the SegNet [41] consists of 26 convolutional layers. These networks have a large number of weight parameters that take a long time to learn and also require long inference time which is crucial as video frames need to be encoded at real-time, hence, making them unsuitable for telementoring applications. In this study, we propose a fast and efficient shallow CNN (S-CNN) architecture for segmentation consisting of four convolution layers that does not perform pixel-wise segmentation, and thus, eliminates the need of a decoder network, unlike state-of-the-art image segmentation networks such as UNet [40] and SegNet [41] that have encoder and decoder networks. The proposed S-CNN is
Fig. 1 Two regions in surgical videos, i.e., the surgical region and background region

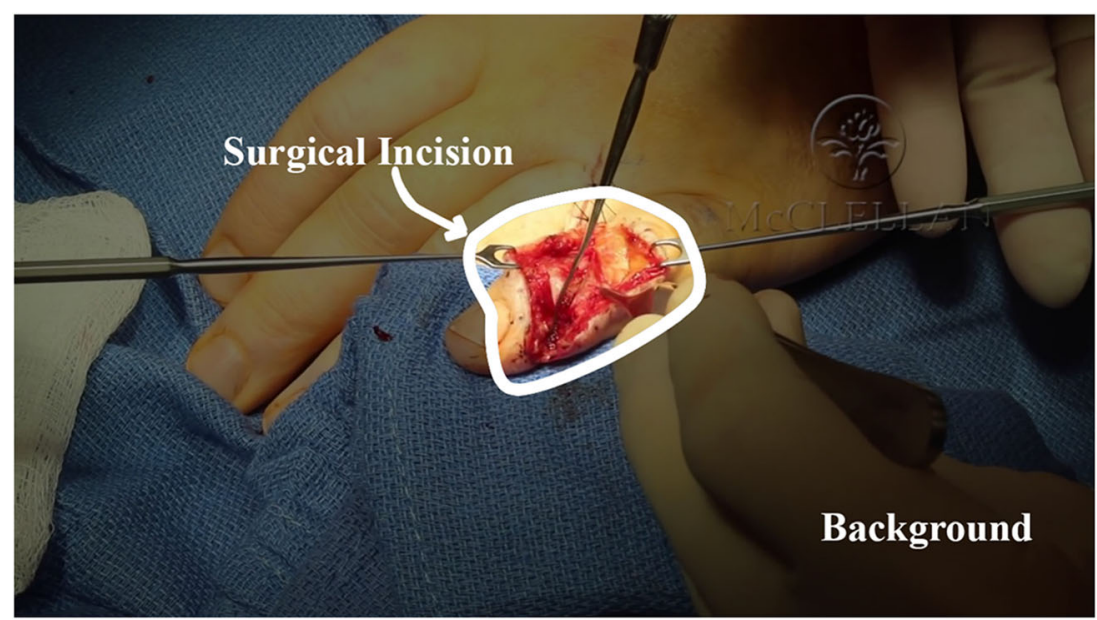


used to segment SR in videos with very less processing time for efficient video compression using HEVC.

In this study, we propose a deep learning (DL)-based HEVC unified framework for telementoring applications, where the quality of SR within the video frames is well preserved, while the number of bits required to code the video sequence is reduced. The objective of this work is to minimize the bandwidth resources required to transmit video, regardless of the other aspects of telecommunications quality-of-service (i.e., packet loss, availability, and transmission delay), which are largely out of the control of the end-user.

The overall system is novel, efficient, and computationally inexpensive that suits real-time video coding for telementoring applications in limited bandwidth scenarios. The performance of the proposed S-CNN-based SR segmentation is compared with mean shift (MST)-based as well as SegNet-based segmentation [41]. The results have shown that the proposed S-CNN performs better than MST and comparably with SegNet, while it is much more computationally efficient. The rest of the paper is organized as follows: Background and Related Work discusses the background and related studies, deep learning Preliminaries discusses deep learning, Proposed S-CNN Architecture for SR Detection discusses the proposed S-CNN-based SR detection, Hybrid Lossless-Lossy HEVC Approach discusses the proposed modification to the HEVC algorithm, Results discusses the experimental results, and finally, and Conclusion concludes.

\section{Background and Related Work}

In recent years, various video coding techniques have been proposed that used different techniques to code certain regions in high-quality and certain regions in low-quality [42-51]. Xu et al. [42] proposed HEVC-based rate control scheme for video conferencing systems to encode facial features in high quality and background regions in lower quality. The authors used the MST algorithm to extract facial features including eyes, nose, and lips. The authors showed that up to $62.8 \%$ encoding time is saved at 1080 pixels resolution with average quality improvement of up to $4.92 \mathrm{~dB}(\mathrm{dBs})$ in the facial region. Similarly, Chai et al. [43] proposed facial regionsbased video coding scheme using HEVC. They proposed two foreground/background bit allocation strategies, which are maximum bit transfer (MBT) and joint bit assignment (JBA). Face segmentation into foreground/background regions was performed using skin-color map-based algorithm. The quality of the foreground (i.e., facial region) was improved, whereas the background image quality was degraded. However, the skin color-based segmentation does not deal with other regions on faces, i.e., hair and beard.

In the field of medical imaging, Gokturk et al. [44] proposed colon computed tomography (CT) image coding system. The region of interest (ROI) in CT was segmented using 3-D morphological image processing technique. Each pixel in the ROI was coded in a lossless manner, and the rest were coded in a lossy manner. Their coding scheme achieved a $2.5 \%$ compression rate at the same level of image quality, exhibiting good results. However, the system was inefficient and needed high bandwidth resources. For similar CT coding, Yu et al. [45] proposed ROI-based rate control for HEVC. The authors performed segmentation of CT and echocardiography video sequence using image processing techniques including Sobel operators, squared gradient, and thresholding. Their scheme achieved bitrate saving of up to $10.64 \%$ and quality improvement of up to $6.95 \mathrm{~dB}$ using H.264 encoder. Other than CT-based coding scheme, Wu et al. [46] used textural image feature-based nearest neighbor (NN) classifier for segmentation in medical ultrasound videos. The authors employed hierarchical coding based on transform coefficient adjustment and a variable quality selection procedure to encode the ultrasound videos. The results showed that on average $13.52 \%$ reduction in bitrate is achieved. However, the NN-based classification was computationally expensive.

A surgical telementoring system was proposed by Khire et al. [47] that provided flexibility of manually selecting different regions in the surgical video for efficient encoding. The authors introduced an interactive ROI selection method, where the video was divided into a $3 \times 3$ grid from which five different locations of ROI could be chosen. These fixed regions include top left, top right, bottom left, bottom right, and central region. The authors coded the selected ROI in high quality and other regions in low quality. Similar to manual interaction scheme, Grois et al. [48] proposed a scalable and adaptive ROI coding scheme using HEVC. The proposed scheme allowed to adaptively set desirable ROI location, size, resolution, and bit-rate, according to the limited network bandwidth and predefined settings. ROI was extracted by removing background layers and then the inter-layer prediction is performed in the extracted region. The experimental results showed that the proposed method improved the coding performance by achieving bitrate savings of up to $33.48 \%$. Similarly, Barsakar et al. [49] proposed ROI coding using kernel-based MST scheme using Huffman encoding. Their method required user interaction to select the ROI and their related resolution using kernel-based MST algorithm. The authors evaluated the technique of CT and magnetic resonance imaging (MRI) videos. The bitrate saved from background regions is adjusted in improving the quality of ROI up to $69.71 \mathrm{~dB}$.

The different ROI-based video coding systems discussed above [47-49] demonstrated good results; however, manually selecting the ROI is a hindrance and causes inconvenience. Instead of manually selecting ROI, Wei et al. [50] proposed a visual saliency-based coding scheme using HEVC. Visual saliency map was used for allocating coding bits or controlling video quality based on the probability of human attention over 
frames. The experimental results showed that $22.52 \%$ of bit rate reduction and $43.48 \%$ encoding time were saved. Similarly, Wong et al. [51] proposed ROI-based channel adaptive video coding scheme. The real-time source video stream is divided into two regions, the ROI and the non-ROI regions. These two regions were encoded adaptively based on the current channel state parameters (data rate), which were fed back to the source coder to adjust the compression ratio.

For ROI-based SR coding scheme, conventional computer vision and machine learning-based segmentation algorithms such as mean shift or k-means can be utilized for SR extraction. Ramya et al. [26] and Wang et al. [27] used MST segmentation for wound boundary determination and wound analysis of foot ulcer in diabetic patients. Similarly, Chang et al. [28] used the MST algorithm to construct wound (foreground) and skin (background) model for real-time wound assessment. Wannous et al. [29] used MST algorithm for wound boundary detection that is used for skin tissue classification. However, such algorithms require manual tuning and manual selection of ROI, which is a hindrance and causes inconvenience for real-time applications. Although the abovementioned techniques can be used to implement surgical telementoring system, they are inefficient and computationally expensive. An automated S-CNN-based SR detection and HEVC-based compression technique will significantly improve the usability of telementoring systems. $\mathrm{CNN}$ is a parallel network of processing nodes that simulates biological learning. Deep learning (DL) consists of a set of techniques that can automatically learn the features from raw data that are useful for image segmentation tasks [52, 53]. The ability to learn representations at multiple levels of abstraction merely by stacking non-linear layers allows DL methods to achieve better generalization on highly complex tasks such as image segmentation. The crucial characteristic of DL approach is that the layers of representations are not hand-engineered by human experts rather discovered from data with a standard learning technique. $\mathrm{CNN}$ is a type of DL method that has recently become modus operandi for image segmentation tasks due to its remarkable achievements in this area [32-35, 53]. This success is partly because of its robust and precise assumptions about the natural images (i.e., locality of associations between pixels and statistical stationarity) [52] and partly due to ease of optimization because of significantly lesser parameters as compared with feed-forward networks [31].

Wang et al. [54] proposed an automatic wound region segmentation and analysis system using CNN. The authors used the learned features of $\mathrm{CNN}$ for healing progress prediction (dates to heal) and infection detection (infected or not). Similarly, Goyal et al. [33] proposed CNN for diabetic foot ulcer (DFU) classification that used parallel convolutions for multiple-level feature extraction. Rajchl et al. [35] proposed DeepCut method for brain and lung segmentation, which learned features using the $\mathrm{CNN}$ model. Similarly, $\mathrm{CNN}$ is also used for understanding images of different domains such as spectral, medical, object and face detection, and multi-class image classification [52, 54-59]. CNN-based DL is a valuable technique that can be used for SR segmentation in telementoring applications.

\section{Deep Learning Preliminaries}

A typical architecture of $\mathrm{CNN}$ for segmentation tasks is composed of convolution, pooling, fully-connected, and sigmoid layers. A short description of these layers is given below:

- Convolution layer: in this layer, each unit is connected to a local patch of units in the previous layer through a set of weights called a filter. The unit activation is called feature map and is computed by applying non-linearity over the locally weighted sums. Rectified linear unit (ReLU) [60] is usually used as an activation function in order to model non-linearity because of its fast convergence [60].

- Pooling layer: while convolution layer learns features, the pooling layer combines semantically related features into a single feature. Each unit in a pooling layer takes input from a patch of units in the previous layer and outputs a maximum or average of these values.

- Full-connected layer: in this layer, each unit is connected to all the units in the previous layer. Typically, the convolution and pooling layer are stacked in two or three stages before using fully-connected layers.

- Sigmoid layer: sigmoid function is used for semantic segmentation tasks where it converts the features into probabilities of each output pixel. This layer contains as many units as the number of pixels of the output image.

In CNN, learning phase deals with optimizing weights of the units with the objective to minimize error. Stochastic gradient descent (SGD) [61] is typically used as an optimization procedure where gradients over the weights are computed by using the standard back-propagation algorithm.

\section{Proposed Telementoring System Using Deep Learning}

The overview of the proposed telementoring system using DL is shown in Fig. 2. In the proposed study, an automated SR segmentation algorithm is proposed that does not depend on manually extracted features or unsupervised approach. Instead, the proposed DL method learns the relevant pictorial features and performs SR segmentation.

The proposed S-CNN model takes raw video frames as input and produces a SR map as output, which is used to encode the surgical video using HEVC. HEVC produces 
Fig. 2 Overview of the proposed telementoring system using deep learning

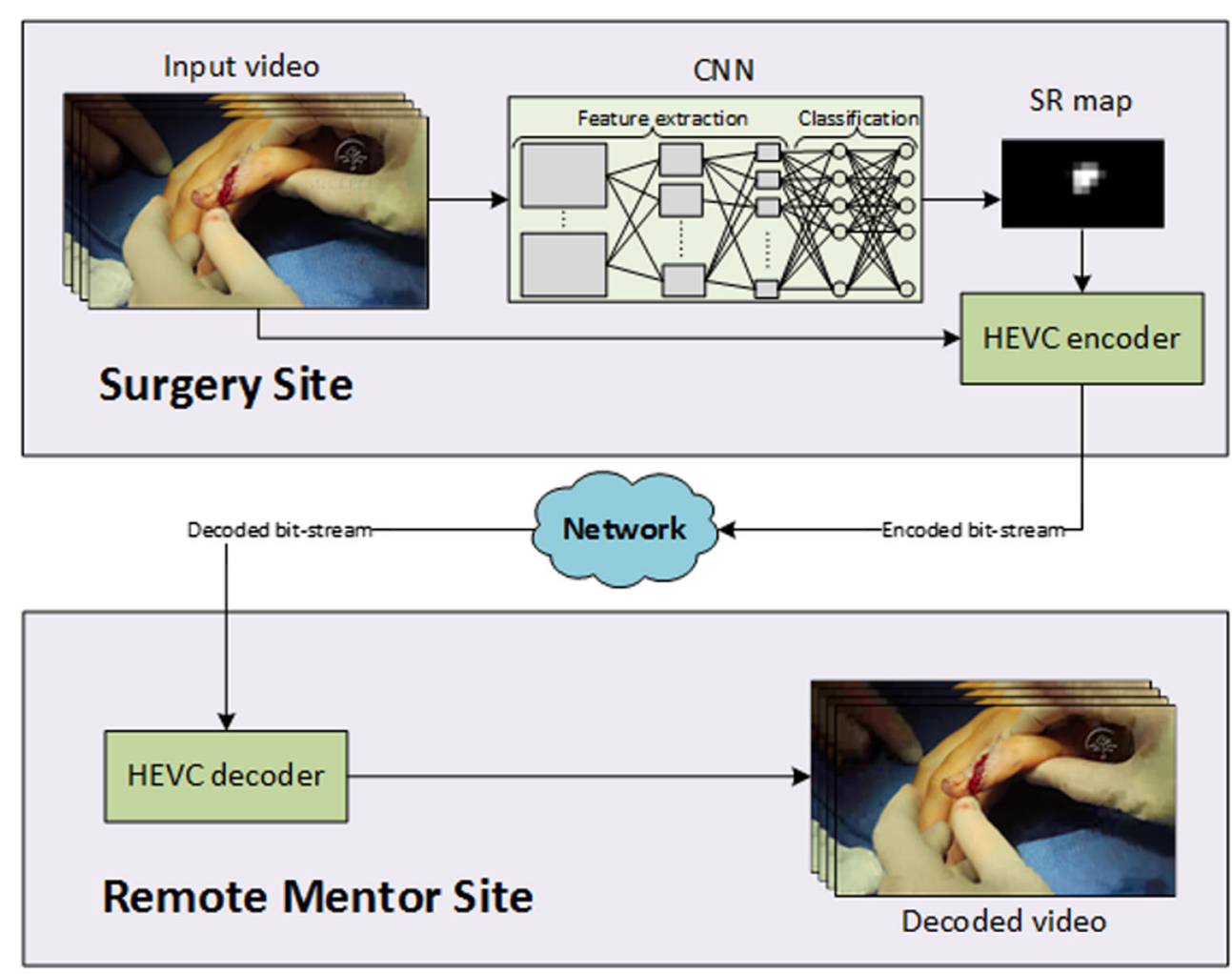

encoded video bitstream which is transmitted over the network to remote mentor site where it is decoded and viewed by a qualified surgeon. The following subsection discusses the proposed DL algorithm.

\section{Proposed S-CNN Architecture for SR Detection}

In this study, the proposed DL algorithm is described as a given input video stream $\left(V_{\text {input }}\right)$ having an $N$ number of frames such that $V=\left\{X_{1}, X_{2}, \ldots, X_{N}\right\}$ where each frame is represented by $X$ is RGB image of height $(H)$ and width $(W)$ such that $X_{N} \in[0 ; 1]^{H \times W \times 3}$; the objective is to learn a function $(f)$ that generates a binary mask $Y \in[0 ; 1]^{r \times c}$, where $r$ and $c$ represent the height and width of the output binary mask, for the ground-truth surgical region $\left(\mathrm{SR}^{\mathrm{gt}}\right)$ :

$f(X) \approx Y$, where $Y=1_{\left\{(r, c) \in \mathrm{SR}^{\mathrm{gt}}\right\}}(X)$.

$\mathrm{CNN}$ is used to learn the function $f$ in an end-to-end style. The proposed S-CNN architecture is created by joining basic computation blocks like convolution, max-pooling, and nonlinear transformation (e.g., ReLU [60]). These stacked operations are grouped into layers to compute network's $i$ th hidden layer activation function $h$ as follows:

$h^{(i)}=\operatorname{pool}\left(\operatorname{ReLU}\left(w^{(i)} * h^{(i-1)}+b^{(i)}\right)\right)$,

where $b^{(i)}$ and $w^{(i)}$ are the bias vector and weight matrix of the $i$ th layer, respectively, and $*$ is the convolution operator, and $h^{(i-1)}$ is either the input image $x$ for $i=1\left(i . e ., h^{(0)}=X\right)$ or $(i-$ $1)$ th hidden layer activation for $i>1$. The output of convolution and max-pooling layers is then flattened and passed through fully connected layers. The output at $k$ th fully connected layer is computed as $g^{(k)}$ as follows:

$g^{(k)}=\operatorname{ReLU}\left(w^{(k)} \cdot g^{(k-1)}+b^{(k)}\right)$,

where (.) indicates dot product and $g^{(k-1)}$ represents either the activation of $k-1^{\text {th }}$ fully connected layer for $k>1$ or $h^{(i)}$ in case of $k=1$. To regularize the network, dropout [31] is used in the training process. The set of $i$ hidden layers and $k$ fully connected layers are stacked together that represent the overall network architecture. The activation layer (i.e., sigmoid) is appended at the end of the network which gives output probability of each neuron between the range of 0 and 1 :

$f(X ; w, b)=\operatorname{sigmoid}\left(g^{(k)} \ldots\left(g^{(2)}\left(g^{(1)}\left(h^{(i)} \ldots\left(h^{(2)}\left(h^{(1)}(X)\right)\right)\right)\right)\right)\right)$,

The final SR map is constructed by applying a threshold of 0.5 on the pixel-wise probabilities:

$\mathrm{SR}= \begin{cases}0, & \text { if } f(X)<0.5 \\ 1, & \text { otherwise }\end{cases}$

Figure 3 shows the proposed S-CNN architecture. In this study, the dimensions $r \times c$ of output mask are set to $12 \times 20$, which is discussed in the next section. 
Fig. 3 Proposed S-CNN architecture

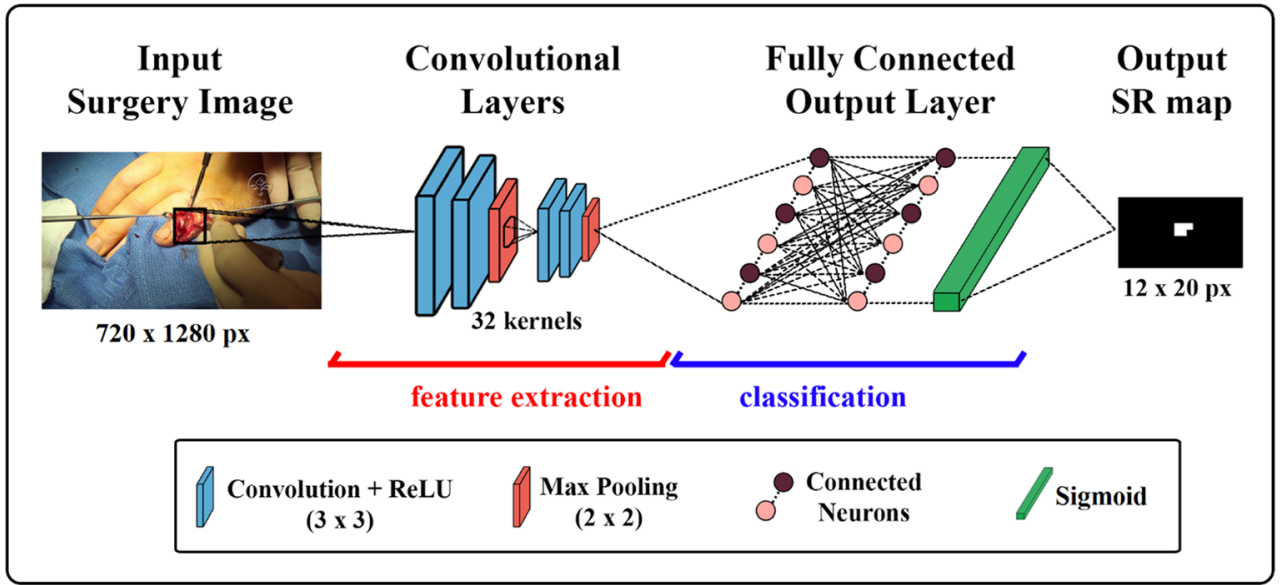

Hybrid Lossless-Lossy HEVC Approach discusses the proposed SR-based HEVC algorithm.

\section{Hybrid Lossless-Lossy HEVC Approach}

HEVC employs a block-based approach to code video sequences using motion prediction and 2-D transforms [5]. Figure 4 shows the main modules of HEVC which are interand intra-prediction, scaling and transformation, quantization, and Context-Adaptive Binary Arithmetic Coding (CABAC) [62]. HEVC splits each video frame into variable sized blocks called Coding Tree Units (CTUs). The CTU size ranges from $16 \times 16$ pixels to $64 \times 64$ pixels and consists of luma $(\mathrm{Y})$ and two chroma ( $\mathrm{Cb}$ and $\mathrm{Cr}$ ) coding tree blocks (CTB). Each CTU is split into coding units (CUs) and each CU can be further partitioned into prediction units (PUs) and transform units (TUs). HEVC makes decisions to perform either inter- or intra-prediction at the CU level. The first frame (I-frame) is coded using intra-prediction. In intra-prediction, information from different regions of the same frame is used for coding. HEVC employs inter-prediction for coding subsequent $\mathrm{P}$ - and B-frames after the initial I-frame. In inter-prediction, information from both subsequent or previous frames can be used for coding. P-frames are coded using forward prediction only, whereas B-frames are coded using both the previous and subsequent frames. After prediction is made, discrete cosine transform (DCT) is used to transform the difference between predictions and the original blocks. The transform coefficients are scaled, quantized, coded, and transmitted along with prediction data.
Fig. 4 Simplified HEVC block diagram

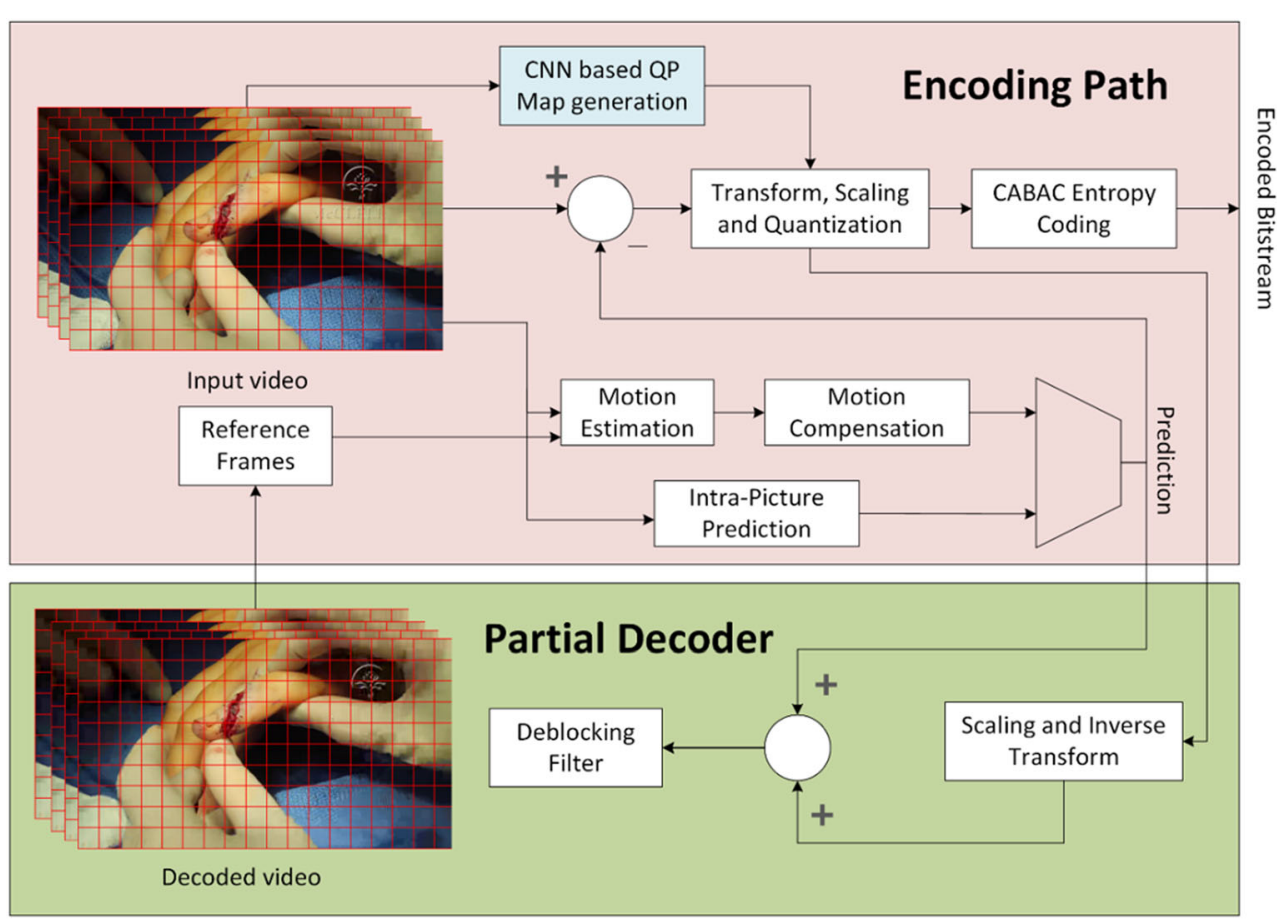


In the proposed methodology, SR-based quality control is introduced where those CTUs that lie in the SR are coded in high quality using low quantization parameter (QP) values, whereas those CTUs that lie in the background region are coded in low quality using high QP values. The QP value for each CTU of a frame is chosen based on a QP map that is created using the SR map as described below. Figure 4 highlights the proposed modification in quantization control of HEVC.

The SR predicted by S-CNN model is used to generate the QP map that gives a measure of distance between SR and background regions. In this study, the size of SR map is chosen based on the maximum CTU size used for encoding (which is $64 \times 64$ pixels) such that each pixel in the SR map corresponds to a single CTU. For example, if input video of size $720 \times 1280$ pixels is divided into CTUs of $64 \times 64$ pixels, it will result in 240 CTUs and the size of the corresponding SR map will be $12 \times 20$ pixels. The distance between the SR and background regions is computed based on the distance transform algorithm proposed by Borgefors [63]. The QP values estimated using these distances are scaled, and a threshold is applied so that distances fall into valid QP range (i.e., 0-51). The scaling factor is chosen such that there is a clear difference visible between SR and background region with a smooth transition. In case SR is not detected, the default $\mathrm{QP}$ value is used to generate the QP map. Each value in the resulting QP map represents QP values that will be assigned to corresponding CTUs in the video frame. The QP map generation algorithm is described as follows:

1. Initialize binary SR map (bin _ SR) by applying thresholds on the S-CNN predicted output.

2. Create a distance map (DM) for SR from the bin _ $S R$ as follows: a. Initialize DM to 0 in $\mathrm{SR}$ region and to three times the maximum value of QP in the background.

b. Compute the distance map in forward and backward passes as follows:

i. Forward pass:

For each row $(r)$ in DM, where $r=2,3, \ldots, m-1$, and $m$ is the number of rows in bin $S R$

For each column $c$ in DM, where $c=2,3, \ldots, n-1$, and $n$ is the number of columns in bin_SR

$\mathrm{DM}_{r, c}$

$=\min \left(\mathrm{DM}_{r-1, c-l}+w_{d}, \mathrm{DM}_{r-1, c}+w_{n}, \mathrm{DM}_{r-1, c+1}+w_{d}, \mathrm{DM}_{r, c-1}+w_{n}, \mathrm{DM}_{r, c}\right)$

End for each

End for each

ii. Backward pass:

For each $r$, where $r=m-1, m-2, \ldots, 2$

For each $c$, where $c=n-1, n-2, \ldots, 2$

$\mathrm{DM}_{r, c}$

$=\min \left(\mathrm{DM}_{r, c}, \mathrm{DM}_{r, c+1}+w_{n}, \mathrm{DM}_{r+1, c-1}+w_{d}, \mathrm{DM}_{r+1, c}+w_{n}, \mathrm{DM}_{r+1, c+1}+w_{d}\right)$

End for each

End for each

Where $w_{d}$ and $w_{n}$ are diagonal- and 4-neighbor weights that are used in the computation of distance at each pixel location.
Fig. 5 Results of the proposed methodology on a sample surgical video frame. a Sample input frame. $\mathbf{b}$ Binary SR map predicted by the S-CNN, which has been scaled-up from its actual size of $12 \times 20$ pixels. c QP map generated using the distance transform algorithm. d The result of the proposed SR-based HEVC scheme. The difference in compression is most visible in the areas outside the SR by examining fine detail

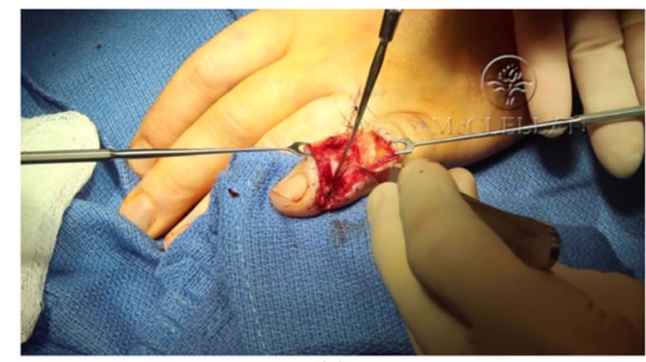

(a)

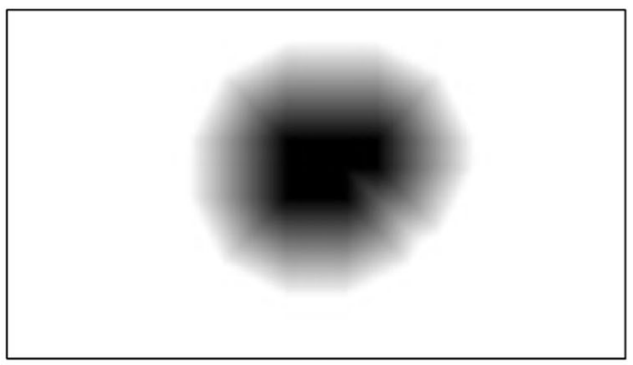

(c)

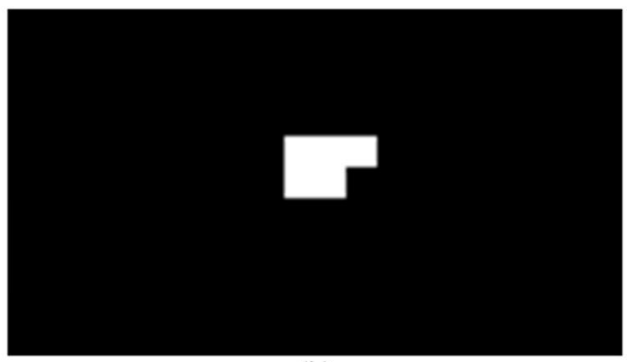

(b)

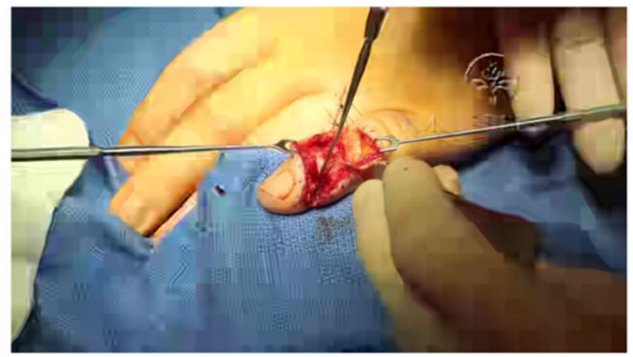

(d) 
Table 1 S-CNN architecture

\begin{tabular}{|c|c|c|c|c|c|c|}
\hline Layer & Layer type & Filter size & No. of filters & Input & Output & Activation \\
\hline L1 & Convolution & $3 \times 3$ & 32 & $3 \times 100 \times 100$ & $32 \times 100 \times 100$ & ReLU \\
\hline L2 & Convolution & $3 \times 3$ & 32 & $32 \times 100 \times 100$ & $32 \times 98 \times 98$ & ReLU \\
\hline L3 & Max-pooling & $2 \times 2$ & - & $32 \times 98 \times 98$ & $32 \times 49 \times 49$ & - \\
\hline L4 & Convolution & $3 \times 3$ & 32 & $32 \times 49 \times 49$ & $32 \times 47 \times 47$ & ReLU \\
\hline L5 & Convolution & $3 \times 3$ & 32 & $32 \times 47 \times 47$ & $32 \times 45 \times 45$ & ReLU \\
\hline L6 & Max-pooling & $2 \times 2$ & - & $32 \times 45 \times 45$ & $32 \times 22 \times 22$ & - \\
\hline L7 & Fully connected & - & - & 15,488 & 512 & ReLU + dropout \\
\hline L8 & Fully connected & - & - & 512 & 240 & Sigmoid \\
\hline
\end{tabular}

3. The distance map (DM) is scaled and then a threshold is applied to create the final QP map:

QP_map $=\left\{\begin{aligned} \mathrm{DM}_{\text {scaled }}, & \text { if } \mathrm{DM}_{\text {scaled }} \leq 51 \\ 51, & \text { otherwise }\end{aligned}\right.$

The generated QP _ map is used by HEVC that encodes the surgical videos and produces the encoded bitstream. Figure 5 shows the results of the proposed SR-based segmentation and encoding algorithm. Figure $5 \mathrm{~b}$ shows the SR map predicted by the S-CNN model, and Fig. 5c shows the QP map that is generated using the SR map. Figure $5 \mathrm{~d}$ shows the result of encoding the frame based on the QP map where the base QP is chosen as 20. As it can be observed, the location of the surgical procedure is visible in high quality, whereas the irrelevant background region is blurry. The performance and results of the proposed system are presented in the Results section.

\section{Results}

In order to evaluate the performance of the proposed methodology, the results are presented in terms of S-CNN training accuracy, segmentation accuracy, and the performance of the proposed SR-based HEVC scheme. Furthermore, the segmentation accuracy of S-CNN is compared with mean shift (MST)-based segmentation [25] and also with the state-ofthe-art image segmentation method referred to as SegNet [41]. The results show that the S-CNN is more accurate as compared with MST and it is comparable with SegNet while being less computationally expensive.

\section{S-CNN Training Accuracy}

The detailed architecture of the proposed S-CNN model is given in Table 1. The model is trained using the Adadelta [64] optimizer with default parameters. The S-CNN model is trained over 50 epochs with a batch size of 25 in Python using Tensorflow [65] libraries. All experiments have been performed on a system with $2.40 \mathrm{GHz}$ Intel Core i5 CPU.

A total of nine surgical videos are used for training the SCNN, which are available online [66-74]. Each video has a resolution of $720 \times 1280$. A total of 1015 frames from these videos were randomly extracted where surgery was in progress, and the SR regions in these videos were manually annotated for training the S-CNN model. The labeling and annotation of the regions in videos are done using domain knowledge and reviewed by the domain expert. In order to avoid overfitting, data augmentation with parameters given in Table 2 was applied to expand the dataset $[35,36]$. Using augmentation, a total of 45,675 frames were generated from the 1015 frames. The augmented dataset was split randomly into training and testing sets with a ratio of $4: 1$. The proposed S-CNN and the utilized SegNet-based SR segmentation model are trained on the augmented training set, and their model accuracy charts are shown in Fig. 6. For SegNet, the open source Tensorflow implementation is used, which is available online at https://github.com/tkuanlun350/Tensorflow-SegNet [41]. The following subsection discusses the segmentation accuracy of the proposed S-CNN model.

\section{SR Segmentation Accuracy}

The SR segmentation accuracy is measured in terms of common segmentation evaluation metrics such as pixel accuracy, mean accuracy, mean Intersection over Union
Table 2 Parameter values for data augmentation

\begin{tabular}{llllllll}
\hline Parameter & $\begin{array}{l}\text { Rotation } \\
\text { range }\end{array}$ & $\begin{array}{l}\text { Width shift } \\
\text { range }\end{array}$ & $\begin{array}{l}\text { Height shift } \\
\text { range }\end{array}$ & $\begin{array}{l}\text { Shear } \\
\text { range }\end{array}$ & $\begin{array}{l}\text { Zoom } \\
\text { range }\end{array}$ & Rescale & $\begin{array}{l}\text { Fill } \\
\text { mode }\end{array}$ \\
\hline Value & 180 & 0.25 & 0.25 & 0.2 & 0.25 & $1 / 255$ & Nearest \\
\hline
\end{tabular}


Fig. 6 The model learning curves. a S-CNN model accuracy. b S-CNN model loss. c SegNet model accuracy. $\mathbf{d}$ SegNet model loss

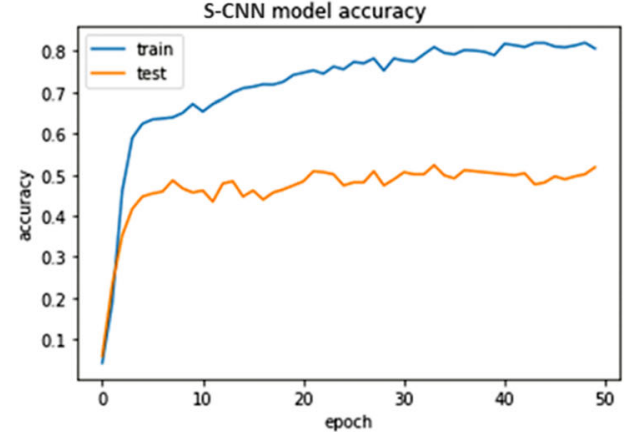

(a)

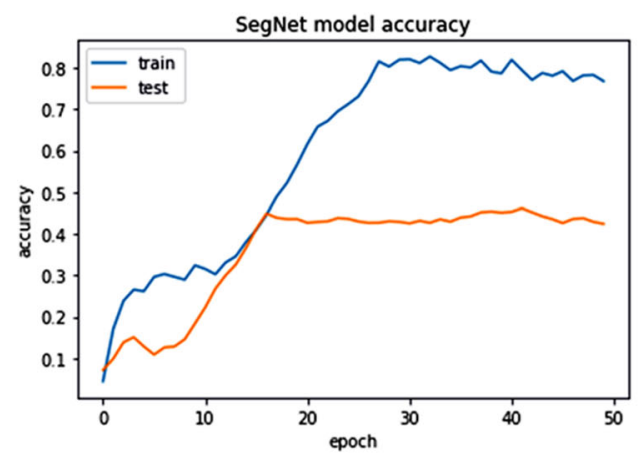

(c)

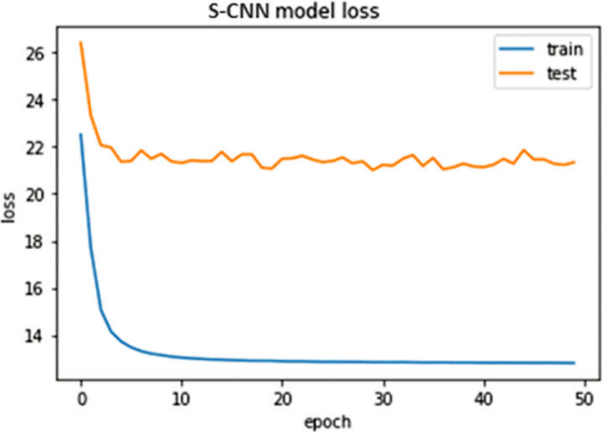

(b)

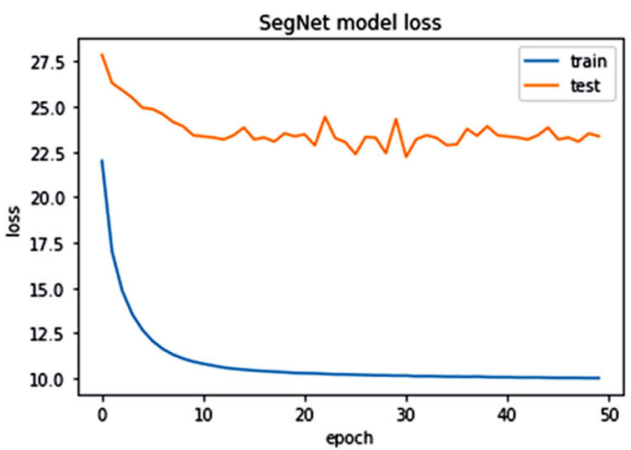

(d)
(IoU), and frequency-weighted (f.w.) IoU [32]. Pixel accuracy indicates the percent of pixels correctly classified in segmentation. Mean accuracy is the average of pixel accuracy for all classes. Mean Intersection over Union indicates the percent of pixels that correctly overlap between the ground truth and the predicted output, whereas frequency weighted Intersection over Union is a more accurate measure of accuracy that takes into account the size of classes. These metrics are computed using Eqs. (912) [32] below, where $n_{i i}$ is the number of pixels correctly identified, $t_{i}$ is the total number of pixels in class $i, n_{c l}$ is the total number of classes, $n_{i j}$ is the number of pixels of class $i$ predicted to belong to class $j$, and $n_{j i}$ is the number of pixels incorrectly rejected for class $i$ :

Pixel accuracy : $\sum_{i} n_{i i} / \sum_{i} t_{i}$
Fig. 7 The process of using the MST algorithm to extract and generate SR map. a Reference input frame of surgery video [66]. b MST segmentation result on the reference frame. $\mathbf{c}$ The extracted surgical region from the segmented frame. d The binary SR map of MST-based SR segmentation

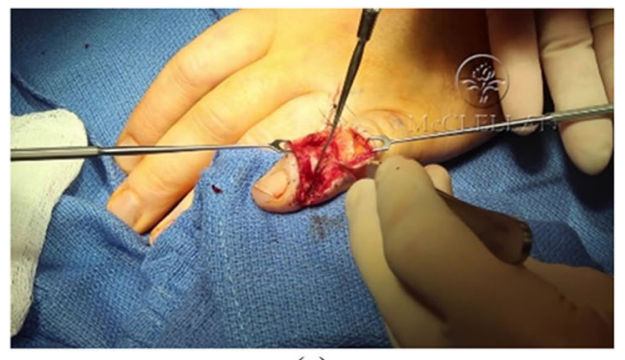

(a)

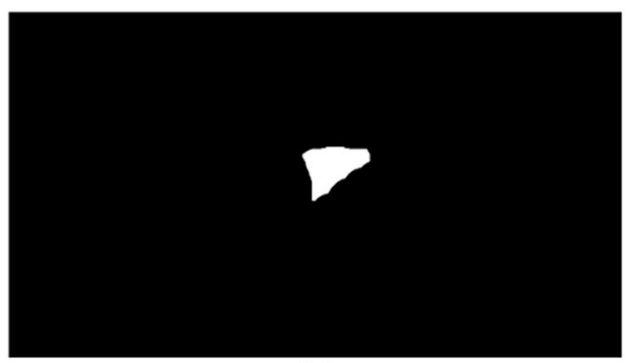

(c)

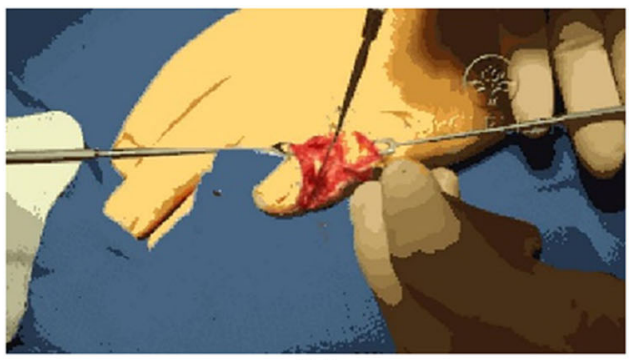

(b)

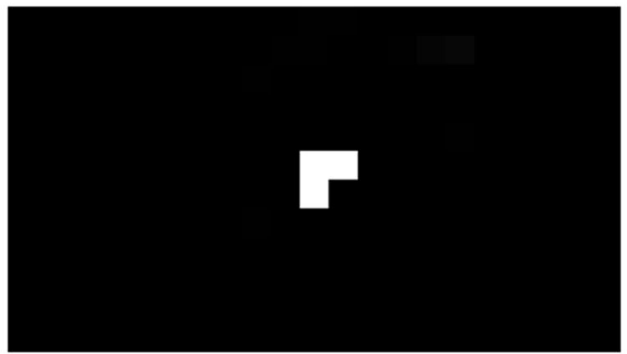

(d) 


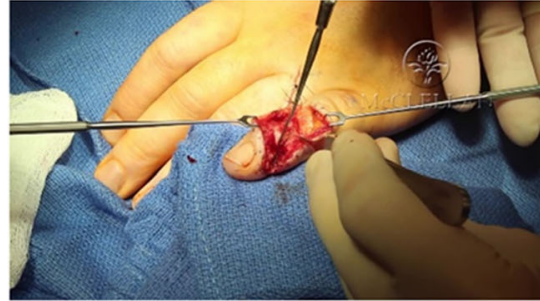

(a)

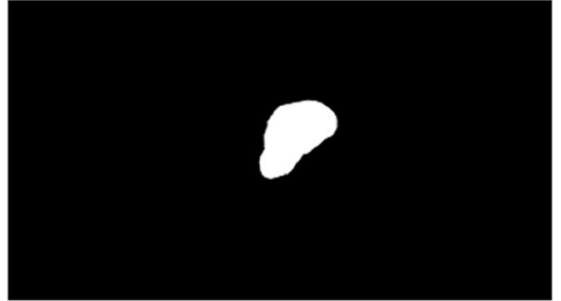

(b)

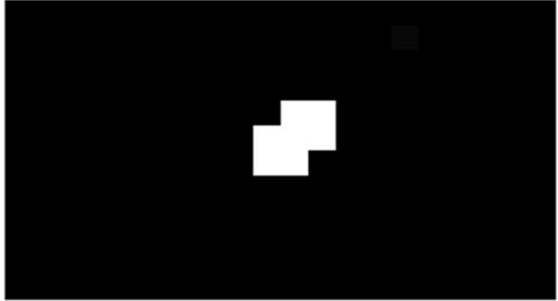

(c)

Fig. 8 The output of SegNet model to segment SR and generated binary SR map. a Reference input frame of surgery video [66]. b SegNet segmentation result on the reference frame. $\mathbf{c}$ The binary SR map using SegNet

Mean accuracy : $\left(1 / n_{c l}\right) \sum_{i} n_{i i} / \sum_{i} t_{i}$

Mean IoU : $\left(1 / n_{c l}\right) \sum_{i} n_{i i} /\left(t_{i}+\sum_{i} n_{j i}-n_{i i}\right)$

f.w.IoU : $\left(\sum_{k} t_{k}\right)^{-1} \sum_{i} t_{i} n_{i i} /\left(t_{i}+\sum_{i} n_{j i}-n_{i i}\right)$

To show the effectiveness of the proposed S-CNNbased segmentation, we have made comparisons with the mean shift (MST) [25] and SegNet-based segmentation methods [41]. For MST segmentation, the open-source implementation available online at https://ww2. mathworks.cn/matlabcentral/fileexchange/52698-k-meansmean-shift-and-normalized-cut-segmentation [75] is used. The MST algorithm was used to segment the input video frames, and the SR was selected by applying a threshold on RGB components of the segmented frame. Figure 7 shows the process of generating SR map using MST segmentation. Figure $7 \mathrm{a}$ shows the reference input frame of Injury [66] video, and Fig. 7b shows its MST-based segmentation result. Figure 7c shows SR map obtained by applying a threshold on RGB component (i.e., red/blood color) of the segmented frame, and Fig. $7 \mathrm{~d}$ shows the binary SR map created using MST-based segmentation. Similarly, the SegNet-based segmentation process is shown in Fig. 8. Figure 8 a shows the reference input frame of Injury video [66], and Fig. 8b shows SegNetbased SR segmentation result. Figure $8 \mathrm{c}$ shows the binary SR map created from the segmented image in Fig. 8b.

The segmentation accuracy results of the S-CNN-, SegNet-, and MST-based segmentation are presented in Table 3. It can be seen that the proposed S-CNN-based SR segmentation achieved high pixel accuracy and mean IoU as compared with MST-based segmentation and shows comparable results with SegNet in all of the evaluated metrics.

The MST segmentation algorithm performs significantly poorly as compared with S-CN- and SegNet-based segmentation because, in some scenarios, it fails to distinguish SR from the skin or other regions such as surgeon's gloves, surgical instruments, and the background due to the similarity in color. This problem with MST segmentation can be observed from Fig. 9, which shows a comparison of binary SR maps generated using MST-, SegNet-, and S-CNN-based segmentation.

Table 3 Comparison of SR segmentation accuracy

\begin{tabular}{|c|c|c|c|c|c|c|c|c|c|c|c|c|}
\hline \multirow[t]{2}{*}{ Video } & \multicolumn{3}{|c|}{ Pixel accuracy (\%) } & \multicolumn{3}{|c|}{ Mean accuracy (\%) } & \multicolumn{3}{|c|}{ Mean IoU (\%) } & \multicolumn{3}{|c|}{ f.w. IoU. (\%) } \\
\hline & MST & SegNet & $\begin{array}{l}\text { S- } \\
\text { CNN }\end{array}$ & MST & SegNet & $\begin{array}{l}\text { S- } \\
\text { CNN }\end{array}$ & MST & SegNet & $\begin{array}{l}\text { S- } \\
\text { CNN }\end{array}$ & MST & SegNet & $\begin{array}{l}\text { S- } \\
\text { CNN }\end{array}$ \\
\hline Injury [66] & 94.3 & 97.1 & 98.1 & 53.5 & 89.4 & 92.9 & 53.5 & 79.1 & 80.1 & 92.2 & 96.9 & 97.2 \\
\hline Cyst [67] & 93.9 & 97.0 & 97.2 & 56.7 & 87.1 & 87.9 & 56.7 & 78.8 & 79.8 & 91.5 & 95.2 & 96.2 \\
\hline Nerve [68] & 96.0 & 97.8 & 98.7 & 49.6 & 86.2 & 90.3 & 49.6 & 77.9 & 79.1 & 94.0 & 97.2 & 97.8 \\
\hline Suture [69] & 95.3 & 98.2 & 97.1 & 51.4 & 92.5 & 92.0 & 51.4 & 79.1 & 78.2 & 92.2 & 98.0 & 97.1 \\
\hline BB [70] & 94.6 & 97.8 & 98.5 & 54.1 & 90.3 & 91.2 & 54.1 & 80.9 & 81.3 & 93.9 & 98.0 & 98.2 \\
\hline Z-Plasty [71] & 94.8 & 97.0 & 98.4 & 52.7 & 88.7 & 89.8 & 52.7 & 79.9 & 80.9 & 92.2 & 98.7 & 97.3 \\
\hline Flexor [72] & 92.5 & 97.1 & 97.0 & 52.6 & 84.2 & 83.7 & 52.6 & 74.4 & 73.2 & 85.9 & 95.2 & 94.7 \\
\hline FDP [73] & 87.7 & 96.1 & 98.7 & 51.7 & 88.2 & 90.0 & 51.7 & 77.3 & 78.4 & 82.7 & 96.2 & 97.9 \\
\hline NuGrip [74] & 95.5 & 97.2 & 98.5 & 49.9 & 86.3 & 87.8 & 49.9 & 78.8 & 80.3 & 92.3 & 96.8 & 97.2 \\
\hline Overall & 94.2 & 96.8 & 97.8 & 53.2 & 88.0 & 89.4 & 53.2 & 77.9 & 79.4 & 91.5 & 95.8 & 96.6 \\
\hline
\end{tabular}




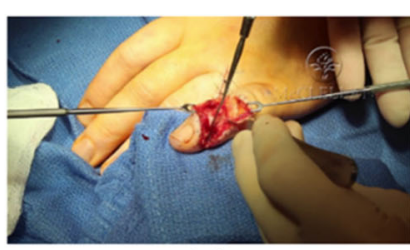

(a)

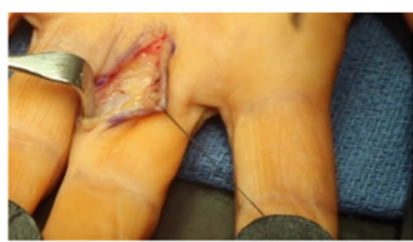

(e)

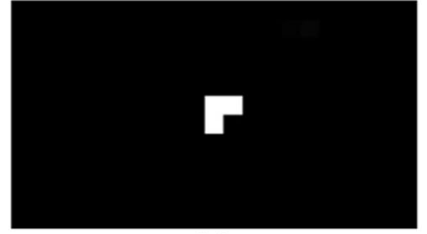

(b)

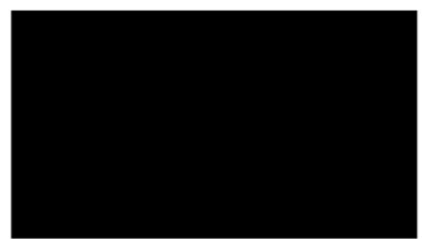

(f)

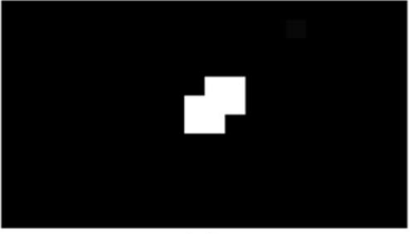

(c)

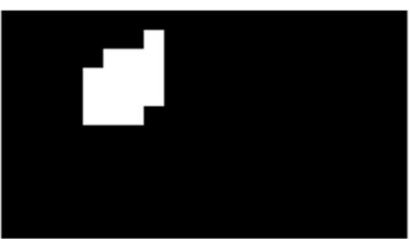

(g)

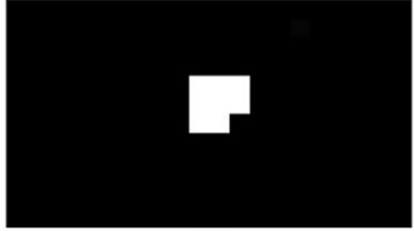

(d)

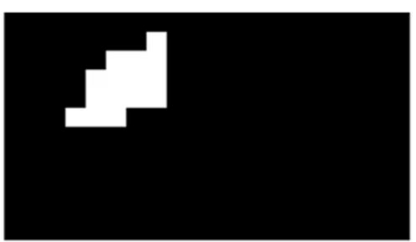

(h)

Fig. 9 Comparison of binary SR map created using MST-, SegNet-, and proposed S-CNN-based segmentation on surgical videos [66, 72]. a The reference frame of Injury [66] video. b, c, and $\mathbf{d}$ Generated binary SR map

Note that the shown binary SR maps are up-scaled for visibility from their original size of $12 \times 20$ pixels. Figure 9 a shows a reference frame from Injury [66] video, its corresponding binary SR maps are shown in Figs. 9b, c, and d generated using MST, SegNet, and proposed S-CNN respectively. It can be seen that the MST segmentation detects smaller SR that does not completely capture the wound region as compared with the S-CNN-based segmentation results. Figure 9e shows a sample frame from Flexor [72] video where the MST fails at detecting the SR, while SegNet and S-CNN accurately detect the SR as shown in Fig. 9f, g, and h respectively.

Furthermore, the computational complexity of the S-CNN is much lesser as compared with MST- and SegNet-based segmentation methods. For MST, many iterations are required for full convergence [76] of clusters for each frame of a video, whereas the S-CNN performs segmentation in a single forward pass through the trained network. The SegNet is computationally expensive due to large numbers of convolution layers and trainable parameters that result in long inference times (26 convolution layers in the encoder and decoder architecture of SegNet), whereas the proposed S-CNN network consists of four convolution layers and a lesser number of trainable parameters as it can be seen from complexity comparison presented in Table 4.

Since the segmentation results are similar for both SegNet- and proposed S-CNN-based SR segmentation, SegNet is not used for comparison of encoding performance in the next section.

of the frame (a) by MST, SegNet, and proposed S-CNN respectively. e The reference frame of Flexor [72] video. $\mathbf{f}, \mathbf{g}$, and $\mathbf{h}$ Generated binary SR map of the frame (e) by MST, SegNet, and proposed S-CNN respectively

\section{SR-Based HEVC Performance}

The HEVC software that is used to carry out the SR-based video coding is the reference HEVC test model (HM 16) (available at https://hevc.hhi.fraunhofer.de/trac/hevc/browser/ tags/HM-16.0). The HEVC performance is measured in terms of bitrate savings and the video quality for both the proposed SR-based encoding and the default full-frame encoding. The Bjøntegaard model $[77,78]$ is one of the most commonly used tools to measure the coding efficiency between two different compression algorithms. Bjøntegaard Delta (BD) metrics are typically measured as a difference in bit rate and video quality (in terms of peak signal-to-noise ratio (PSNR)), which is computed using the rate-distortion (R-D) curves. The R-D curve given by a set of $M$ bit rate values $\left(B R_{1}, B R_{2}, \ldots, B R_{M}\right)$ with corresponding PSNR measurements $\left(D N_{1}, D N_{2}, \ldots, D N_{M}\right)$ is approximated as follows $[77,78]$ :

$\widehat{\mathrm{DN}}(\mathrm{br})=w \mathrm{br}^{3}+x \mathrm{br}^{2}+y \mathrm{br}+z$

where $\hat{\mathrm{DN}}$ is the fitted distortion in PSNR, br is the logarithm of bit rate, i.e., $\mathrm{br}=\log \mathrm{BR}$ and $w, x, y$, and $z$ are the parameters of the fitting function. The video compression quality performance is measured in terms of the average Bjøntegaard delta peak signal-to-noise ratio (BD-PSNR), which is approximated by the difference between the integrals of the fitted R-D curves divided by the integration interval $[77,78]$ :
Table 4 Comparison of the network complexities in terms of total number of trainable parameters

\begin{tabular}{lllll}
\hline Network & Input & Output & Convolutional layers & Trainable parameters \\
\hline S-CNN & $3 \times 100 \times 100$ & $1 \times 12 \times 20$ & 4 (encoder) & 8.08 million \\
SegNet [41] & $3 \times 64 \times 64$ & $1 \times 64 \times 64$ & 26 (encoder + decoder) & 29.5 million \\
\hline
\end{tabular}


Table 5 HEVC parameters used for experimentation

\begin{tabular}{ll}
\hline Parameters & Values \\
\hline Max CU size & $64 \times 64$ \\
Max CU partition depth & 3 \\
Number of frames & 120 \\
Frames per second & 24 \\
\hline
\end{tabular}

$\mathrm{BD}-\mathrm{PSNR}=\frac{1}{\mathrm{br}_{b}-\mathrm{br}_{a}} \int_{\mathrm{br}_{a}}^{\mathrm{br}_{b}}\left[\widehat{\mathrm{DN}}_{2}(\mathrm{br})-\widehat{\mathrm{DN}}_{1}(\mathrm{br})\right] d \mathrm{br}$

where $\hat{\mathrm{DN}}_{1}(\mathrm{br})$ and $\hat{\mathrm{DN}}_{2}(\mathrm{br})$ are two fitted R-D curves, and $\mathrm{br}_{a}$ is minimum value and $\mathrm{br}_{b}$ is the maximum value of integration bounds respectively. Similarly, to express the rate as a function of the distortion, and to approximate the R-D values $[77,78]$ :

$\widehat{\mathrm{br}}(\mathrm{DN})=w \mathrm{DN}^{3}+x \mathrm{DN}^{2}+y \mathrm{DN}+z$

The bit rate difference is measured using Bjontegaard delta bit rate (BD-BR), which is the average bit rate difference between two R-D curves approximated as [77, 78]:

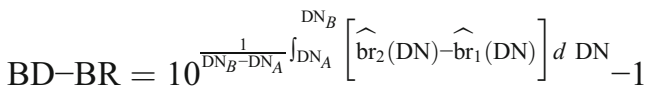

where $\hat{\mathrm{br}}_{1}(\mathrm{DN})$ and $\hat{\mathrm{br}}_{2}(\mathrm{DN})$ are the two fitted R-D curves, and $\mathrm{DN}_{A}$ is minimum value and $\mathrm{DN}_{B}$ is the maximum value of integration bounds respectively. In our scenario, the R-D curves of default HEVC encoding and the proposed SR- based HEVC encoding is compared and results are carried out using discussed Bjontegaard delta metric [77, 78]. The HEVC encoding parameters that are used to carry out the comparisons are given in Table 5.

Table 6 presents comparison results between the proposed SR-based HEVC and the default full-frame HEVC on Injury [66] video. The QP values are divided into three quality ranges, with an increment step size of 5: high (QPs 0 to 20), medium (QPs 20 to 35), and low (QPs 35 to 51). It can be observed from Table 6 that the proposed algorithm achieved a significant BD-BR reduction of $88.8 \%$ in high-quality settings while maintaining the PSNR in SRs of the default and the proposed encoding. The PSNR comparison in Table 6 is only made for the SR since the background region is unimportant for telementoring applications, whereas the reduction in overall encoded file size is very important. The improvement in BD-PSNR for SR came out to be $11.5 \mathrm{~dB}$ in comparison with default HEVC encoding at high-quality setting. These results are also shown graphically in Fig. 10, which shows that high bitrate saving is achieved in a high-quality setting.

Figure 11 shows sample frames for comparison of output video quality when the surgical video is encoded with both the default HEVC and the proposed SR-based encoding at low-, medium-, and high-quality settings. By varying the quality settings, the effect of the SR and QP map-based encoding can be observed. At medium- and high-quality settings, there is a clear difference between SR and background regions. As the video quality setting is increased, the region of high quality around the SR expands, and it can be observed in Fig. 11a-d.

Table 7 presents video encoding results based on SR extracted using the proposed S-CNN- and MST-based
Table 6 Comparison of BD-BR and BD-PSNR between default HEVC and proposed methodology on video [66]

\begin{tabular}{|c|c|c|c|c|c|c|}
\hline \multirow[t]{2}{*}{ QP } & \multirow{2}{*}{$\begin{array}{l}\text { Default HM } \\
\text { bitrate }(\mathrm{kb} / \mathrm{s})\end{array}$} & \multirow{2}{*}{$\begin{array}{l}\text { Proposed } \\
\text { methodology bitrate } \\
(\mathrm{kb} / \mathrm{s})\end{array}$} & \multirow{2}{*}{$\begin{array}{l}\text { Average } \Delta \\
\text { BD-BR }(\%)\end{array}$} & \multicolumn{2}{|c|}{ Average PSNR in SR } & \multirow{2}{*}{$\begin{array}{l}\text { Average } \Delta \text { BD- } \\
\text { PSNR (dB) }\end{array}$} \\
\hline & & & & $\begin{array}{l}\text { Default } \\
\text { HEVC }\end{array}$ & $\begin{array}{l}\text { Proposed } \\
\text { method }\end{array}$ & \\
\hline \multicolumn{7}{|c|}{ High-quality setting } \\
\hline 0 & $45,697.71$ & 5372.79 & & 64.07 & 64.12 & \\
\hline 5 & $28,201.38$ & 2936.11 & & 56.17 & 56.18 & \\
\hline 10 & $13,240.36$ & 1502.49 & -88.76 & 52.83 & 52.84 & 11.49 \\
\hline 15 & 6619.81 & 797.71 & & 49.85 & 49.85 & \\
\hline 20 & 3088.86 & 430.88 & & 47.18 & 47.16 & \\
\hline \multicolumn{7}{|c|}{ Medium-quality setting } \\
\hline 25 & 1446.76 & 246.91 & & 44.31 & 44.30 & \\
\hline 30 & 669.91 & 145.85 & -77.86 & 41.55 & 41.49 & 6.97 \\
\hline 35 & 325.71 & 89.83 & & 38.57 & 38.42 & \\
\hline \multicolumn{7}{|c|}{ Low-quality setting } \\
\hline 40 & 166.18 & 60.72 & & 35.45 & 35.31 & \\
\hline 45 & 83.67 & 45.81 & -41.72 & 32.34 & 32.22 & 2.77 \\
\hline 51 & 38.70 & 38.70 & & 29.02 & 29.02 & \\
\hline
\end{tabular}


Fig. 10 Comparison of bitrate and PSNR at QP values between the default HEVC and the proposed SR-based encodings on Injury [66] video

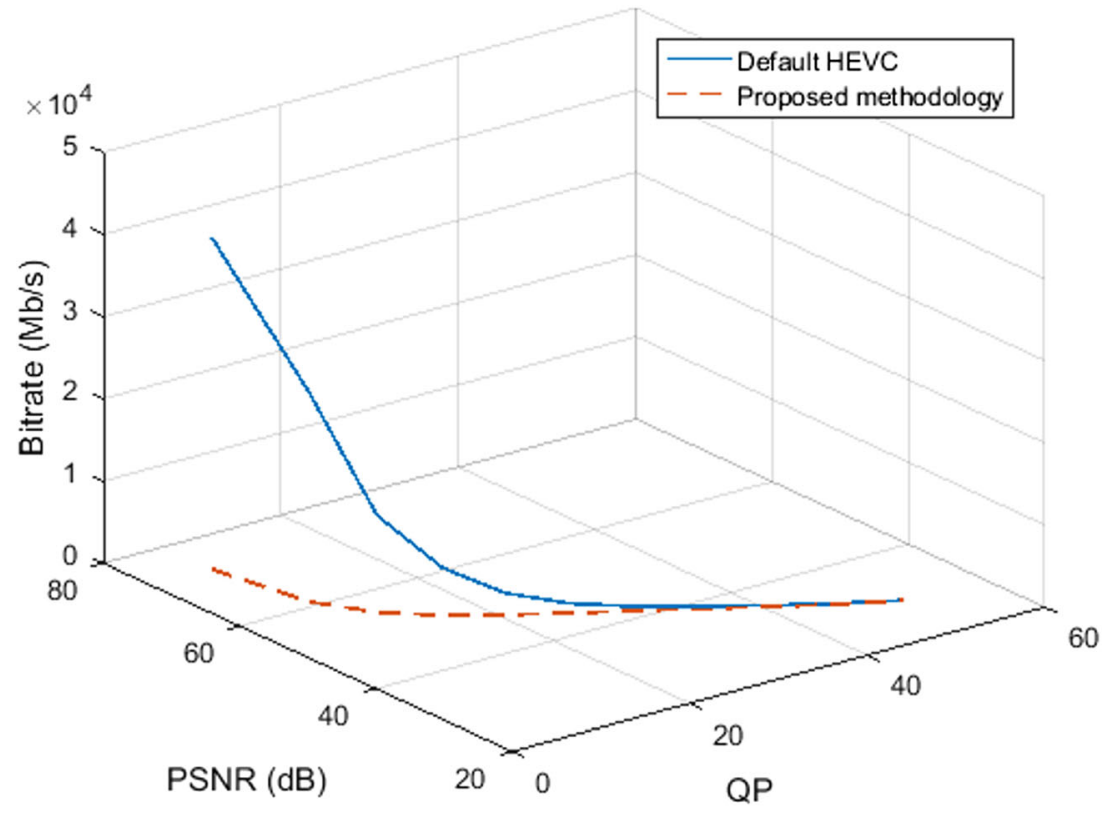

segmentation on other surgical videos [66-74] mentioned in Table 3. The results are compared based on overall bitrate savings and the visual quality in SR measured in terms of PSNR. For simplicity, a single base QP of 20 is chosen to encode the surgical videos based on the analysis presented in Table 6, which shows that using QPs in range of 0-20 results in high bitrate savings without loss in video quality in the SR. It can be seen from Table 7 that although the MST-based SR coding scheme achieved high bitrate savings ( 88.15 to $98.40 \%$ ), the visual quality in SR is badly affected (7.89 to $16.91 \mathrm{~dB}$ loss as compared with average PSNR in SR using default full-frame encoding), whereas the proposed S-CNN-based SR coding scheme shows high quality in SR that is comparable with the default HEVC encoding while achieving good bitrate saving $(74.71 \%$ to $86.05 \%)$.

A pictorial comparison of HEVC encoded frames based on SR extracted using S-CNN and MST segmentation is shown in Fig. 12. Figures 12a and b show reference frames of Nerve [68] video encoded using MST- and S-CNN-based segmentation respectively. It is clearly visible in Fig. 12a that MST-based SR encoding resulted in

Fig. 11 Comparison of reference frame quality in injury [66] video at different QPs. a Encoded using base QP of 0 . b Encoded using base QP of 20. c Encoded using base QP of 30. d Encoded with base QP of 45

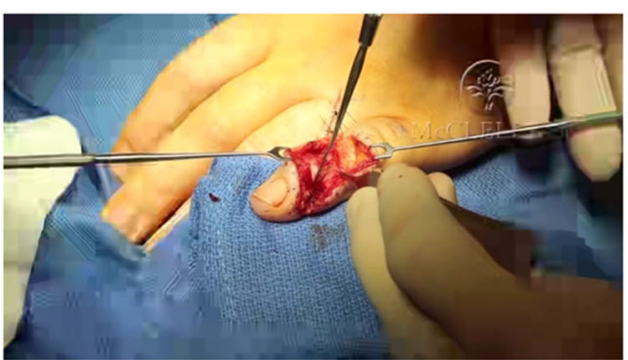

(a)

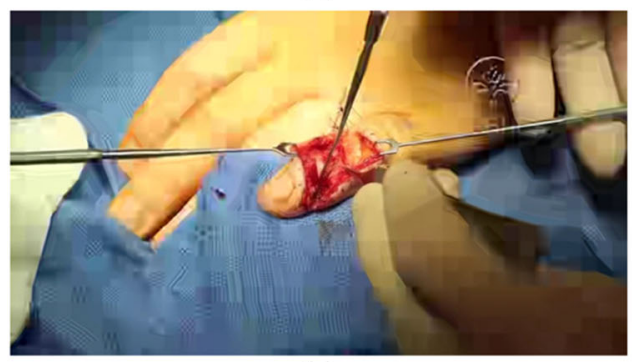

(c)

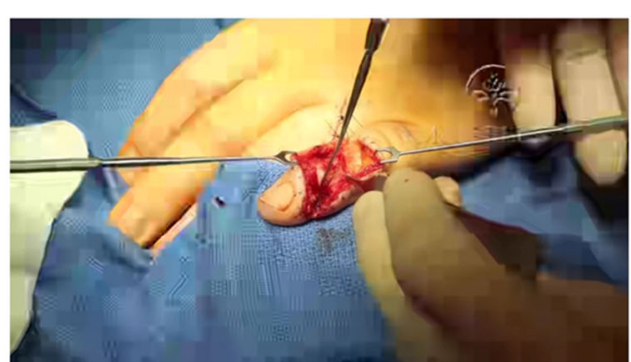

(b)

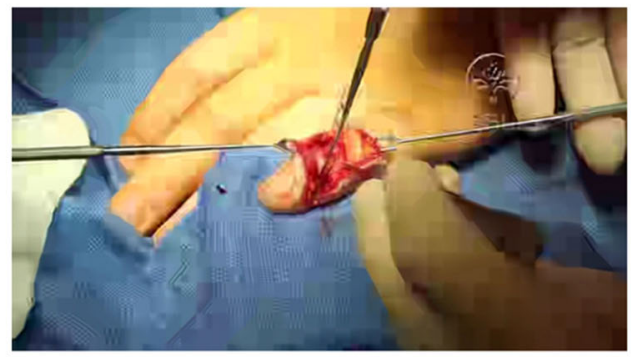

(d) 
Table 7 Bitrate savings and PSNR difference of proposed algorithm for different videos encoded at base QP of 20

\begin{tabular}{|c|c|c|c|c|c|c|c|c|}
\hline \multirow[t]{2}{*}{ Video } & \multicolumn{3}{|c|}{ Bitrate (kb/s) } & \multicolumn{2}{|c|}{ Bitrate savings (\%) } & \multicolumn{3}{|c|}{ Average PSNR in SR } \\
\hline & Default & MST & $\mathrm{S}-\mathrm{CNN}$ & MST & S-CNN & Default & MST & $\mathrm{S}-\mathrm{CNN}$ \\
\hline Injury [66] & 3088.86 & 192.18 & 430.88 & 93.78 & 86.05 & 47.18 & 40.04 & 47.16 \\
\hline Cyst [67] & 2875.16 & 136.39 & 574.33 & 95.26 & 80.02 & 45.13 & 34.15 & 45.09 \\
\hline Nerve [68] & 2234.07 & 75.76 & 524.38 & 96.61 & 76.53 & 46.63 & 30.72 & 46.57 \\
\hline Suture [69] & 5043.00 & 80.54 & 980.96 & 98.40 & 80.55 & 47.73 & 31.17 & 47.71 \\
\hline $\mathrm{BB}[70]$ & 1614.94 & 191.39 & 277.10 & 88.15 & 82.84 & 44.90 & 33.91 & 44.79 \\
\hline Z-Plasty [71] & 1776.08 & 176.79 & 446.53 & 90.05 & 74.86 & 45.74 & 37.85 & 45.69 \\
\hline Flexor [72] & 2435.43 & 248.83 & 608.50 & 89.78 & 75.02 & 47.56 & 38.91 & 47.52 \\
\hline FDP [73] & 2393.51 & 277.22 & 868.80 & 88.41 & 63.70 & 44.73 & 34.19 & 44.72 \\
\hline NuGrip [74] & 2096.84 & 85.31 & 530.22 & 95.93 & 74.71 & 44.86 & 30.03 & 44.81 \\
\hline
\end{tabular}

poor visual quality in the surgery region, whereas Fig. 12b shows that high visual quality is achieved in SR using proposed S-CNN-based segmentation. Similarly, Fig. 12c and d show encoded reference frames of FDP [73] video using both schemes respectively. In Fig. 12c, the SR extracted using MST-based coding scheme is small that adversely affects visual quality, whereas the proposed S-CNN-based encoding scheme shows gradual quality distribution between SR and background region as shown in Fig. 12d. These results demonstrate that the proposed S-CNN-based SR encoding scheme is very effective in reducing the required bandwidth resources while maintaining high visual quality in SR, thus enabling remote mentors to effectively supervise surgical procedures in limited bandwidth scenarios.

\section{Conclusion}

This paper introduced a surgical region-based quality selective HEVC method for efficiently transmitting surgical videos over limited bandwidth networks. SR in the surgical video was predicted using the proposed S-CNN. The SR map and the source frame were given as input to the modified HEVC algorithm that assigned low QP values to SR, whereas high QP values were assigned to unimportant background regions. The performance of the proposed S-CNN based SR segmentation is compared with mean shift (MST)-based as well as SegNet-based segmentation [41]. The proposed S-CNN model accurately segmented SRs in nine test surgical videos as pixel accuracy of $97 \%$, mean accuracy of $89 \%$, mean IoU accuracy of $79 \%$, and f.w mean IoU of $96 \%$ were achieved. Similarly, experimental results showed that on average, the
Fig. 12 The result of encoding reference frames of video $[68,73]$ using base QP of 20. a and $\mathbf{c}$ Result of MST-based SR coding on the reference frame of Nerve [68] and FDP [73] videos respectively. $\mathbf{b}$ and $\mathbf{d}$ Result of proposed S-CNN-based SR coding on the reference frame of Nerve [68] and FDP [73] videos respectively

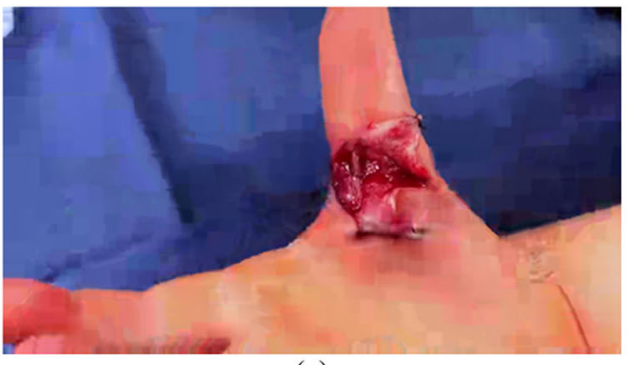

(a)

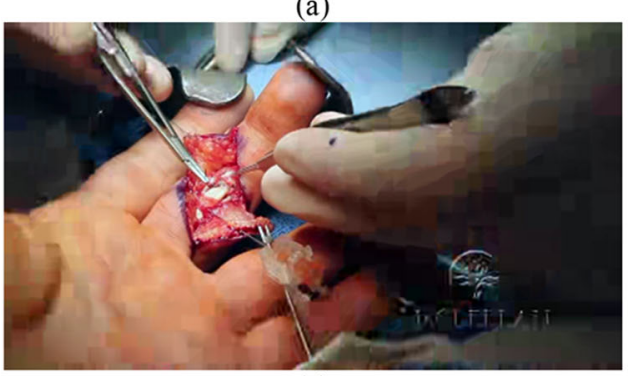

(c)

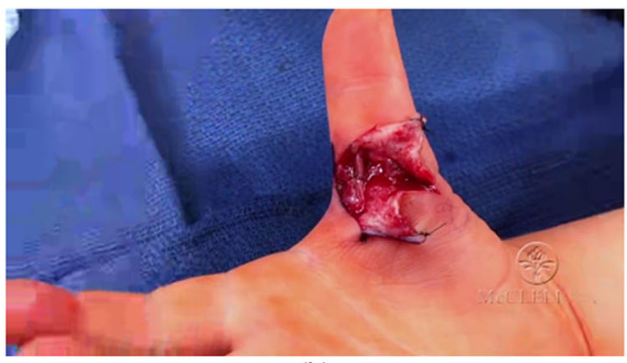

(b)

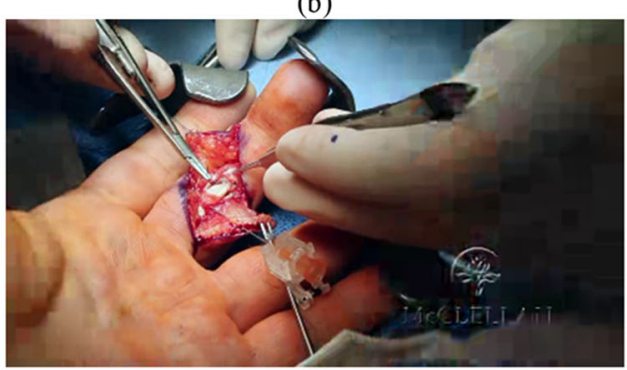

(d) 
proposed SR-based algorithm demonstrated significant BDBR reduction of $88.8 \%$ at high-quality settings (QP in range of 0-20) as compared with default full-frame HEVC encoding. The BD-PSNR gain in SR at the same QP setting came out to be $11.5 \mathrm{~dB}$. To make a fair comparison of the proposed scheme with other similar optimal bit allocation scheme, we have used the mean shift segmentation algorithm to extract SR and performed SR-based video coding using HEVC. The MST-based SR coding scheme achieved high bitrate savings ( 88.15 to $98.40 \%$ ), the visual quality in SR is badly affected (7.89 to $16.91 \mathrm{~dB}$ loss as compared with average SR-PSNR in default full-frame encoding), whereas the proposed S-CNN-based SR coding scheme shows high quality in SR that is comparable with the default HEVC encoding while achieving good bitrate saving (74.71 to $86.05 \%$ ). Based on these results, the proposed S-CNN-based SR encoding scheme can be considered a highly efficient solution for limited bandwidth telementoring applications.

Acknowledgments The authors acknowledge that the surgical procedure videos of Dr. Thomas McClellan [66-73] and Dr. Vangelisti MD [74] are used for experimentation in this research.

\section{References}

1. Ereso AQ, Garcia P, Tseng E, Gauger G, Kim H, Dua MM, Guy TS: Live transference of surgical subspecialty skills using telerobotic proctoring to remote general surgeons. J Am Coll Surg 211(3): 400-411, 2010

2. Poropatich R, Lappan C, Lam D: Operational Use of US Army Telemedicine Information Systems in Iraq and Afghanistan Considerations for NATO Operations, Telemedicine for Trauma, Emergencies, and Disaster Management, Artech House, 173-182, 2010

3. Rosser JC, Young SM, Klonsky J: Telementoring: an application whose time has come. Surg Endosc 21(8):1458-1463, 2007

4. Rayman R, Croome K, Galbraith N, McClure R, Morady R, Peterson S, Primak S: Robotic telesurgery: a real-world comparison of ground-and satellite-based internet performance. Int J Med Robot Comput Assisted Surg 3(2):111-116, 2007

5. Sullivan GJ, Ohm J, Han WJ, Wiegand T: Overview of the high efficiency video coding (HEVC) standard. IEEE Trans Circuits Syst Video Technol 22(12):1649-1668, 2012

6. Ostermann J, Bormans J, List P, Marpe D, Narroschke M, Pereira F, Wedi T: Video coding with H. 264/AVC: tools, performance, and complexity. IEEE Circuits Syst Mag 4(1):7-28, 2004

7. Nemcic O, Vranjes M, Rimac-Drlje S: Comparison of H. 264/AVC and MPEG-4 Part 2 coded video. IEEE, In ELMAR, 41-44, 2007

8. Marpe D, Wiegand T, Sullivan GJ: The H. 264/MPEG4 advanced video coding standard and its applications. IEEE Commun Mag 44(8):134-143, 2006

9. Kim H, Rhee CE, Lee HJ: A low-power video recording system with multiple operation modes for H. 264 and light-weight compression. IEEE Trans Multimedia 18(4):603-613, 2016

10. Ogawa K, Ohtake G: Watermarking for HEVC/H. 265 stream. In Consumer Electronics (ICCE), IEEE International Conference, 102-103, 2015
11. Antoniou ZC, Panayides AS, Pantziaris M, Constantinides AG, Pattichis CS, Pattichis MS: Real-time adaptation to time-varying constraints for medical video communications. IEEE J Biomed Health Informat:2017

12. Doyle TE, Musson DM, Schwering T: Cognitive priority model for advanced telemedical support in Limited Bandwidth Applications. In Electrical and Computer Engineering (CCECE), IEEE 30th Canadian Conference, 1-6, 2017

13. Panayides AS, Pattichis MS, Constantinides AG, Pattichis CS: Mhealth medical video communication systems: an overview of design approaches and recent advances. In Engineering in Medicine and Biology Society (EMBC), 35th Annual International Conference of the IEEE, 7253-7256, 2013

14. Sanchez V, Bartrina-Rapesta J: Lossless compression of medical images based on HEVC intra coding. In Acoustics, Speech and Signal Processing (ICASSP), IEEE International Conference, 6622-6626, 2014

15. Rad RM, Saeedi P, Bajic I: Automatic cleavage detection in H. 264 sequence of human embryo development. In Electrical and Computer Engineering (CCECE), IEEE Canadian Conference, 14, 2016

16. Neri A, Battisti F, Carli M, Salatino M, Goffredo M, D’Alessio T: Perceptually lossless ultrasound video coding for telemedicine applications. In Proc. Int. workshop video process. Quality Metrics, 2007

17. Tahir M, Ul-Abdin Z, Qadir MA: Enhancing the HEVC video analyzer for medical diagnostic videos. In High-Capacity Optical Networks and Enabling/Emerging Technologies (HONET), 12th International Conference, 1-5, 2015

18. Jangbari P, Patel D: Review on region of interest coding techniques for medical image compression. Int J Comput Appl (0975-8887) 134(10):1-5, 2016

19. Ul-Abdin Z, Shafique M, Qadir MA: Telemedicine Aware Video Coding Under Very-Low Bitrates. International Conference on Health Informatics and Medical Systems, HIMS'16, 130-136, 2016

20. He J, Yang F: Efficient frame-level bit allocation algorithm for $\mathrm{H}$. 265/HEVC. IET Image Process 11(4):245-257, 2017

21. Chien WD, Liao KY, Yang JF: H. 264-based hierarchical two-layer lossless video coding method. IET Signal Process 8(1):21-29, 2014

22. Diaz R, Blinstein S, Qu S: Integrating HEVC Video Compression with a High Dynamic Range Video Pipeline. SMPTE Motion Imaging J 125(1):14-21, 2016

23. Pham DL, $\mathrm{Xu} \mathrm{C}$, Prince JL: Current methods in medical image segmentation. Annu Rev Biomed Eng 2(1):315-337, 2000

24. Dhanachandra N, Chanu YJ: A survey on image segmentation methods using clustering techniques. Eur J Eng Res Sci 2(1):1520, 2017

25. Comaniciu D, Meer P: Mean shift: a robust approach toward feature space analysis. IEEE Trans Pattern Anal Mach Intell 24(5):603619, May 2002

26. Ramya R, Jenitta A: Foot injury detection using K-means clustering and mean shift segmentation algorithm. Int J Adv Res Basic Eng Sci Technol (IJARBEST) 3(24):323-329, 2017

27. Wang L, Pedersen PC, Strong DM, Tulu B, Agu E, Ignotz R: Smartphone-based wound assessment system for patients with diabetes. IEEE Trans Biomed Eng 62(2):477-488, 2015

28. Chang MC, Yu T, Luo J, Duan K, Tu P, Zhao Y, Nagraj N, Rajiv V, Priebe M, Wood EA, Stachura M: Multimodal sensor system for pressure ulcer wound assessment and care. IEEE Trans Ind Informat 14(3):1186-1196, 2018

29. Wannous H, Treuillet S, Lucas Y: Robust tissue classification for reproducible wound assessment in telemedicine environments. J Electron Imaging 19(2):023002, 2010

30. Nilakant R, Menon HP, Vikram K: A survey on advanced segmentation techniques for brain MRI image segmentation. Int J Adv Sci Eng Inf Technol 7(4):1448-1456, 2017 
31. Krizhevsky A, Sutskever I, Hinton GE: Imagenet classification with deep convolutional neural networks. In Advances in neural information processing systems, 1097-1105, 2012

32. Long J, Shelhamer E, Darrell T: Fully convolutional networks for semantic segmentation. In Proceedings of the IEEE conference on computer vision and pattern recognition, 3431-3440, 2015

33. Goyal M, Reeves ND, Davison AK, Rajbhandari S, Spragg J, Yap MH: DFUNet: Convolutional Neural Networks for Diabetic Foot Ulcer Classification. arXiv preprint arXiv:1711.10448, 2017

34. Badea MS, Felea II, Florea LM, Vertan C: The use of deep learning in image segmentation, classification and detection. arXiv preprint arXiv:1605.09612, 2016

35. Rajchl M, Lee MC, Oktay O, Kamnitsas K, Passerat-Palmbach J, Bai W, Rueckert D: DeepCut: object segmentation from bounding box annotations using convolutional neural networks. IEEE Trans Med Imaging 36(2):674-683, 2017

36. Chaichulee S, Villarroel M, Jorge J, Arteta C, Green G, McCormick K, Tarassenko L: Multi-task Convolutional Neural Network for Patient Detection and Skin Segmentation in Continuous Noncontact Vital Sign Monitoring. In Automatic Face and Gesture Recognition (FG 2017), 12th IEEE International Conference, 266-272, 2017

37. Simonyan K, Zisserman A: Very deep convolutional networks for large-scale image recognition. arXiv preprint arXiv:1409.1556, 2014

38. Szegedy C, Liu W, Jia Y, Sermanet P, Reed S, Anguelov D, Erhan D, Vanhoucke V, Rabinovich A: Going deeper with convolutions. In Proceedings of the IEEE conference on computer vision and pattern recognition, 1-9, 2015

39. He K, Zhang X, Ren S, Sun J: Deep residual learning for image recognition. In Proceedings of the IEEE conference on computer vision and pattern recognition, 770-778, 2016

40. Ronneberger O, Fischer P, Brox T: U-net: Convolutional networks for biomedical image segmentation. In: International Conference on Medical image computing and computer-assisted intervention. Cham: Springer, 2015, pp. 234-241

41. Badrinarayanan V, Kendall A, Cipolla R: Segnet: A deep convolutional encoder-decoder architecture for image segmentation. arXiv preprint arXiv:1511.00561, 2015

42. Xu M, Deng X, Li S, Wang Z: Region-of-interest based conversational HEVC coding with hierarchical perception model of face. IEEE J Sel Top Sign Process 8(3):475-489, 2014

43. Chai D, Ngan KN, Bouzerdoum A: Foreground/background bit allocation for region-of-interest coding. In Image Processing, Proceedings. IEEE International Conference on September 2000, 2:923-926, 2000

44. Gokturk SB, Tomasi C, Girod B, Beaulieu C: Medical image compression based on region of interest, with application to colon $\mathrm{CT}$ images. In Engineering in Medicine and Biology Society, 2001. Proceedings of the 23rd Annual International Conference of the IEEE, 3:2453-2456, 2001

45. Yu H, Lin Z, Pan F: Applications and improvement of H. 264 in medical video compression. IEEE Trans Circuits Syst I Reg Pap 52(12):2707-2716, 2005

46. Wu Y, Liu P, Gao Y, Jia K: Medical ultrasound video coding with $\mathrm{H}$. 265/HEVC based on ROI extraction. PLoS One 11(11):e0165698, 2016

47. Khire S, Robertson S, Jayant N, Wood EA, Stachura ME, Goksel T: Region-of-interest video coding for enabling surgical telementoring in low-bandwidth scenarios. In Military Communications Conference, 2012-Milcom, 1-6, 2012

48. Grois D, Kaminsky E, Hadar O: ROI adaptive scalable video coding for limited bandwidth wireless networks. In Wireless Days (WD), October 2010 IFIP, 1-5. IEEE, 2010

49. Barsakar T, Mankar V: A novel approach for medical video compression using kernel based meanshift ROI coding techniques. In
Advances in Signal Processing (CASP), Conference, IEEE , 212 216, 2016

50. Wei H, Zhou X, Zhou W, Yan C, Duan Z, Shan N: Visual saliency based perceptual video coding in HEVC. In Circuits and Systems (ISCAS), IEEE International Symposium, 2547-2550, 2016

51. Wong ACW, Kwok YK: On a region-of-interest based approach to robust wireless video transmission. In Parallel Architectures, Algorithms and Networks, May 2004. Proceedings. 7th International Symposium, IEEE, 385-390, 2004

52. LeCun Y, Bengio Y, Hinton G: Deep learning. Nature 521(7553): 436, 2015

53. Litjens G, Kooi T, Bejnordi BE, Setio AAA, Ciompi F, Ghafoorian M, Van Der Laak JA, Van Ginneken B, Sánchez CI: A survey on deep learning in medical image analysis. Med Image Anal 42:6088, 2017

54. Wang C, Yan X, Smith M, Kochhar K, Rubin M, Warren SM, Lee $\mathrm{H}$ : A unified framework for automatic wound segmentation and analysis with deep convolutional neural networks. In Engineering in Medicine and Biology Society (EMBC), 37th Annual International Conference of the IEEE, 2415-2418, 2015

55. Shenoy VN, Foster E, Aalami L, Majeed B, Aalami O: Deepwound: Automated Postoperative Wound Assessment and Surgical Site Surveillance through Convolutional Neural Networks. In IEEE International Conference on Bioinformatics and Biomedicine (BIBM), 1017-1021, 2018

56. Zeiler MD, Fergus R: Visualizing and understanding convolutional networks. In: European conference on computer vision. Cham: Springer, 2014, pp. 818-833

57. Anthimopoulos M, Christodoulidis S, Ebner L, Christe A, Mougiakakou S: Lung pattern classification for interstitial lung diseases using a deep convolutional neural network. IEEE Trans Med Imaging 35(5):1207-1216, 2016

58. Shin HC, Roth HR, Gao M, Lu L, Xu Z, Nogues I, Summers RM: Deep convolutional neural networks for computer-aided detection: $\mathrm{CNN}$ architectures, dataset characteristics and transfer learning. IEEE Trans Med Imaging 35(5):1285-1298, 2016

59. Oliveira GL, Bollen C, Burgard W, Brox T: Efficient and robust deep networks for semantic segmentation. Int J Robot Res 0278364917710542, 2017

60. Nair V, Hinton GE: Rectified linear units improve restricted boltzmann machines. In Proceedings of the 27th International Conference on Machine Learning (ICML-10), 807-814, 2010

61. Bottou L: Stochastic gradient descent tricks. In: Neural networks: tricks of the trade. Berlin: Springer, 2012, pp. 421-436

62. Sze V, Budagavi M: High throughput CABAC entropy coding in HEVC. IEEE Trans Circuits Syst Video Technol 22(12):1778 1791, 2012

63. Borgefors G: Distance transformations in digital images. Comput Vis Graph Image Process 34(3):344-371, 1986

64. Zeiler MD: ADADELTA: an adaptive learning rate method. arXiv preprint arXiv:1212.5701, 2012

65. Abadi M, Barham P, Chen J, Chen Z, Davis A, Dean J, Devin M, Ghemawat S, Irving G, Isard M, Kudlur M: Tensorflow: a system for large-scale machine learning. In OSDI, 16:265-283, 2016

66. McClellan T: Live Surgery: Small Finger Extensor Tendon Saw Injury Cut Repair. Youtube, Available from: https://youtu.be/ 3o7cgZsd3bs. Accessed 30.10.17

67. McClellan T: Live Surgery: Ganglion Cyst Volar Wrist. Youtube, Available from: https://youtu.be/ZgNJ8YDA7dY. Accessed 25.10. 17

68. McClellan T: The digital nerve was cut! The wrinkle test worked and helped child. Youtube, Available from: https://youtu.be/ CY1HYIBrAwQ. Accessed 05.11.17

69. McClellan T: Live Surgery: Running Subcuticular Suture, What is an Intracuticular or Subcuticular Suture?, Youtube, Available from: https://youtu.be/CiW93U-3XcQ. Accessed 10.11.17 
70. McClellan T: Live Surgery: Foreign Body (BB) Removal from Finger. Youtube, Available from: https://youtu.be/ DWQ6WX3ImBU. Accessed 25.10.17

71. McClellan T: Live Surgery: Z-Plasty of Scar Contracture (Finger). Youtube, Available from: https://youtu.be/wdseg3UvXrI. Accessed 25.10 .17

72. McClellan T: Live Surgery: Ganglion Cyst: Flexor Tendon Sheath (Finger). Youtube, Available from: https://youtu.be/hDZBE8tcctE. Accessed 25.10.17

73. McClellan T: Live Surgery: Flexor Digitorum Profundus (FDP) Finger Tendon Repair). Youtube, Available from: https://youtu.be/ boMlEa3P43g. Accessed 25.10.17

74. Vangelisti MD: Hand Surgery Procedure - NuGrip Arthroplasty (Thumb Arthritis Joint Replacement Surgery). Youtube, Available from: https://youtu.be/YZgDQ15kWFs. Accessed 25.10.17

75. Asvadi A: K-means, Mean-shift and Normalized-cut segmentation. MathWorks, Available from: https://ww2.mathworks.cn/ matlabcentral/fileexchange/52698-k-means-mean-shift-andnormalized-cut-segmentation. Accessed 05.01.19

76. Carreira-Perpinán MA: A review of mean-shift algorithms for clustering. arXiv preprint arXiv:1503.00687, 2015

77. Bjntegaard G: Calculation of average PSNR differences between RD-curves (VCEG-M33). In VCEG meeting (ITU-T SG16 Q. 6), 2001

78. Hanhart P, Ebrahimi T: Calculation of average coding efficiency based on subjective quality scores. J Vis Commun Image Represent 25(3):555-564, 2014

Publisher's Note Springer Nature remains neutral with regard to jurisdictional claims in published maps and institutional affiliations. 